OPEN

SUBJECT AREAS:

SOLAR ENERGY AND

PHOTOVOLTAIC

TECHNOLOGY

SILICON PHOTONICS

Received

20 June 2014

Accepted

15 August 2014

Published

4 September 2014

Correspondence and requests for materials should be addressed to F.A.M. (fredrik. martinsen@ntnu.no)

\section{Silicon-core glass fibres as microwire radial-junction solar cells}

\author{
F. A. Martinsen', B. K. Smeltzer' , M. Nord', T. Hawkins², J. Ballato² \& U. J. Gibson'
}

'Department of Physics, Norwegian University of Science and Technology, N-7491, Trondheim, Norway, ${ }^{2}$ The Center for Optical Materials Science and Engineering, Technologies (COMSET), Department of Material Science and Engineering, Clemson, SC, 29634, USA.

Vertically aligned radial-junction solar cell designs offer potential improvements over planar geometries, as carrier generation occurs close to the junction for all absorption depths, but most production methods still require a single crystal substrate. Here, we report on the fabrication of such solar cells from polycrystalline, low purity (99.98\%) p-type silicon starting material, formed into silicon core, silica sheath fibres using bulk glass draw techniques. Short segments were cut from the fibres, and the silica was etched from one side, which exposed the core and formed a conical cavity around it. We then used vapour deposition techniques to create $\mathrm{p}$-i-n junction solar cells. Prototype cells formed from single fibres have shown conversion efficiencies up to $3.6 \%$, despite the low purity of the starting material. This fabrication method has the potential to reduce the energy cost and the silicon volume required for solar cell production. Simulations were performed to investigate the potential of the conical cavity around the silicon core for light collection. Absorption of over $90 \%$ of the incident light was predicted, over a wide range of wavelengths, using these structures in combination with a $10 \%$ volume fraction of silicon.

$\mathrm{n}$ the quest for greater utilisation of solar photovoltaic conversion, many avenues are being pursued to reduce costs, improve performance, or take advantage of abundant, low toxicity constituent materials. One approach to reduce the cost and improve the performance is the use of vertically aligned radial junction solar cells. Radial junctions increase absorption and decouple the carrier collection path from the optical absorption direction by aligning most of the junction with the direction of the impinging light. The design, usually consisting of a forest of free-standing wires, makes use of the natural light trapping of this geometry in order to increase the amount of light absorbed per semiconductor volume. Radial junction cells have been realized from a wide range of materials, including GaAs, InP, InGaAs and dye sensitized oxides, as well as organic and chalcogenide fibres ${ }^{1-8}$. However, silicon still dominates the solar cell market, and improvements within this established technology area are of interest.

Vertically aligned silicon radial junction solar cells are being investigated as an alternative to planar silicon wafers to reduce the cost of silicon solar cells while maintaining high efficiency ${ }^{9-20}$. The radius of the semiconductor microwire can be matched to the minority carrier diffusion length ${ }^{21}$, allowing efficient modules to be fabricated from lower quality materials ${ }^{16,22}$. However, for very high aspect-ratio wires, surface recombination, and long distances for majority carrier extraction can be problematic. For optimum performance, microwire height should be on the order of 100-200 $\mu \mathrm{m}$ and the diameter should be close to the minority carrier recombination length ${ }^{21}$, which may be up to tens or hundreds of microns depending on silicon quality. The desired scale is thus one that is readily accessible via the bulk, commercially-employed fibre-drawing technique used here. In addition, purification during drawing is possible, as the melting and recrystallization of the silicon during the drawing process leads to in-situ segregation of impurities -- on a timescale that is much less than that needed when bulk silicon is processed ${ }^{23,24}$ due to the small diameter of the fibre core.

Radial-junction silicon solar cells have been successfully produced using the catalyst assisted vapour liquid solid (VLS) growth method ${ }^{9-14}$ and deep reactive ion etching of silicon wafers ${ }^{15-17}$. Both methods have produced cells with efficiencies of $\sim 10 \%$, as well as significantly enhanced light absorption per unit of silicon compared to planar cells ${ }^{9,16}$. Nonetheless, the reliance on single-crystal substrates as well as introduction of detrimental impurities during production for both methods motivates the search for alternative approaches. One such route is the fabrication of silicon-core glass-clad fibres, with a subsequent assembly of a substrate-independent cell, which may also allow new flexible designs to be realized. The fibre cores are the only silicon in the design, potentially reducing the amounts of material and energy required for solar cell production. 
Two known methods for fabrication of free-standing silicon fibres are high-pressure chemical vapour deposition (HPCVD) in capillary pores ${ }^{25}$ and molten-core fibre drawing ${ }^{26-28}$. He, et al. ${ }^{25}$ demonstrated the HPCVD approach for solar applications, where they made $15 \mu \mathrm{m}$ diameter flexible coaxial in-fibre solar cells with conversion efficiencies of $0.5 \%$. Early molten-core fibres were reported to suffer from mechanical stress as well as oxygen incorporation ${ }^{26}$, and were not investigated earlier as a potential solar cell material. Recently, the use of alkaline earth oxide interface modifiers ${ }^{29,30}$ to relieve mechanical stress and hinder oxygen in-diffusion during production of long silicon core fibres was demonstrated. This modification was used here in production of fibres from low purity silicon, and we report on their performance as radial junction solar cells. The drawing process improved the purity of the fibres, and etching of the interface layer also led to formation of a conical cavity in the silica cladding surrounding the silicon cores. The potential of this conical cavity for light trapping was investigated through simulations.

A heterojunction with intrinsic thin layer (HIT) $\operatorname{design}^{31-34}$ was chosen for initial fabrication of fibre-based solar cells. The HITdesign, developed in the 1990s by Sanyo (now Panasonic), utilizes a layer of amorphous hydrogenated intrinsic silicon (a-i-Si:H), instead of the more common $\mathrm{SiO}_{2}{ }^{11}$ or $\mathrm{Si}_{\mathrm{x}} \mathrm{N}_{\mathrm{y}}{ }^{14,35}$ for surface passivation, with an amorphous doped silicon layer on top acting as the emitter. The a-i-Si:H passivates dangling bonds at the surface of the crystalline core $\mathrm{e}^{20,36}$, and the higher bandgap of amorphous silicon results in the formation of a majority carrier mirror, enhancing carrier collection ${ }^{36,37}$.

\section{Results}

Silicon-core fibres. The silicon-core fibres were produced with a range of diameters, varied by changing the preform feed rate (1$3 \mathrm{~mm} / \mathrm{min}$ ) and drawing speed (2-3 m/min). As shown in Fig. 1, the silicon fibre core was surrounded by an interfacial layer and a silica cladding, and analysis with electron dispersive $\mathrm{x}$-ray spectroscopy (EDS) showed that the calcium oxide, pre-deposited on the inside of the silica preform, was confined to a thin interface layer. Calcium oxide is known for the bulk "slag-refining" of silicon where it serves as a sink for impurities, and in fibres the calcium oxide and silica also formed a eutectic structure that accommodated strain

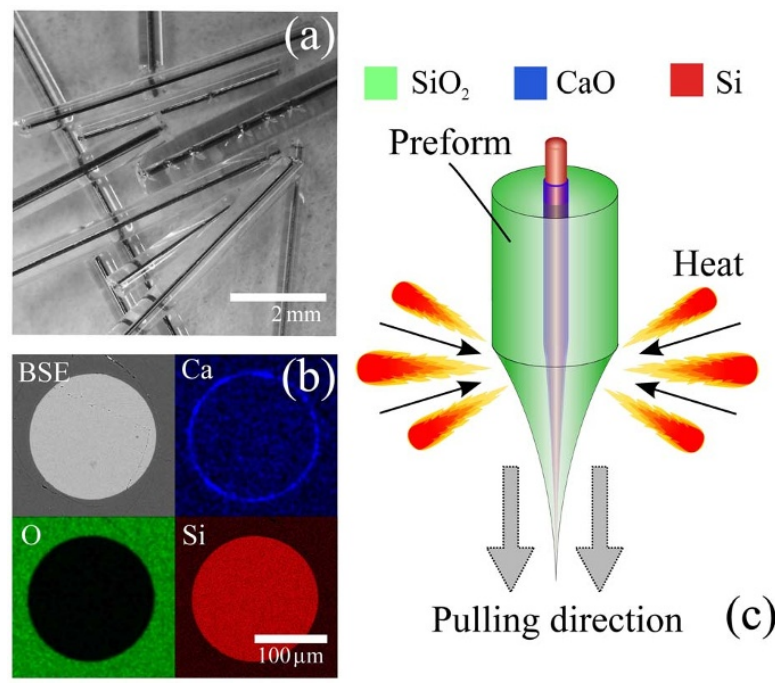

Figure $1 \mid$ The fibre product and fabrication method. (a) photograph of fibre pieces with various diameters, (b) a backscattered electron micrograph and energy dispersive $\mathrm{x}$-ray spectroscopy maps for calcium, silicon and oxygen of a fibre cross-section, and (c) schematic of fibre structure during pulling. The silicon core and silica cladding are separated by a thin Ca-containing interface layer. as it solidified. Segregation of transition metals and other impurities have been observed during rapid solidification of flakes made with $98 \%$ pure silicon ${ }^{24}$, and a similar reduction of impurities in the fibres reported here was suggested by large bulk carrier diffusion lengths (see discussion). The p-type fibres had a resistivity of $0.3-0.9 \Omega-\mathrm{cm}$, similar to the silicon starting material, and oxygen levels were measured to be $1.3 \times 10^{17}$ and $2.1 \times 10^{17}$ atoms $/ \mathrm{cm}^{3}$ for the starting material and the fibre respectively, demonstrating that the silicon is not significantly degraded by the drawing process. Without the interface layer, oxygen levels greater than $5 \times 10^{21}$ atoms $/ \mathrm{cm}^{3}$ were reported ${ }^{26}$. The core of our fibres was crystalline silicon with typical grain lengths of several millimetres, as determined by x-ray and electron back scattered diffraction (EBSD). This was larger than the height of the solar cells made from the fibres, so grain boundaries were not incorporated into most cells, despite the rapid solidification of the silicon. Thus the drawing and recrystallization processes led to silicon that was sufficiently pure and had sufficiently large grains to be used for solar cell production, even when starting with a lowpurity material.

Single fibre solar cells. Single fibre radial junction solar cells with an open circuit voltage of $0.3 \mathrm{~V}$, a short-circuit current density of $16.6 \mathrm{~mA} / \mathrm{cm}^{2}$, a fill factor of 0.52 , and an overall efficiency (using the silicon cross- sectional area as the reference illuminated area) of $3.6 \%$ were fabricated. The silicon area was used as we were primarily interested in assessing the quality of the silicon made by fibre drawing. Including additional area would reduce the overall efficiency, but as we show in simulations below, the glass may contribute to performance in future devices. Fig. 2 presents the fabrication procedure, where fibres initially were cut into short segments (Fig. 2a) and one side of these segments was etched in hydrofluoric (HF) acid. The acid attacked the silica cladding and the interface modifier, and a conical cavity (etch cone) formed around the fibre due to rapid etching of the calcium-containing layer (Fig. 2b,f). A second etch was performed on the silicon core in order to remove some of the material damaged by the slicing process as well as to reduce the roughness of the fibre surface (Fig. 2g). Intrinsic and n-type amorphous silicon and electrical contacts were vapour deposited and the I-V characteristics were then measured. Fig. 2e presents the I-V curves of one of the cells under dark and illuminated conditions, both for an as-made cell (Fig. 2h) and for the same cell where the conical cavity surrounding the fibre core was filled with an opaque conductive paste (Fig. 2i). The filling was performed to assess the resistive losses incurred by the use of indium tin oxide (ITO) as the sole conducting path on the top surface, and the potentially poor contact at the bottom of the cavity. Measurement of the as-made cell resulted in similar open circuit voltage and short circuit current as for the filled case, but with a reduction of fill factor from 0.52 down to 0.43 . For the filled case, the measured cell can be considered as a single free-standing silicon fibre where the incident light is absorbed only through the top cross section of the fibre.

Amorphous silicon layers. A planar witness cross section transmission electron microscopy (TEM)-sample was prepared in order to inspect a representative interface and the thicknesses of the amorphous layer. Scanning transmission electron microscopy (STEM), electron energy loss spectroscopy (EELS) and EDS were used to examine this sample. As shown in Fig. 3, the amorphous silicon layers had a total thickness of $\sim 32 \mathrm{~nm}$. The outer edge of the film was determined by increasing the brightness of the STEM image, but the original is presented here to allow visualization of the silicon lattice. The ratio of the deposition times for the intrinsic and doped layers was 2:7, in agreement with the measured phosphorus profile, giving layer thicknesses of $\approx 7 \mathrm{~nm}$ and $\approx 25 \mathrm{~nm}$ respectively. These represented the maximum thicknesses possible in the device, since reduced flow of reactants as well as reduced 


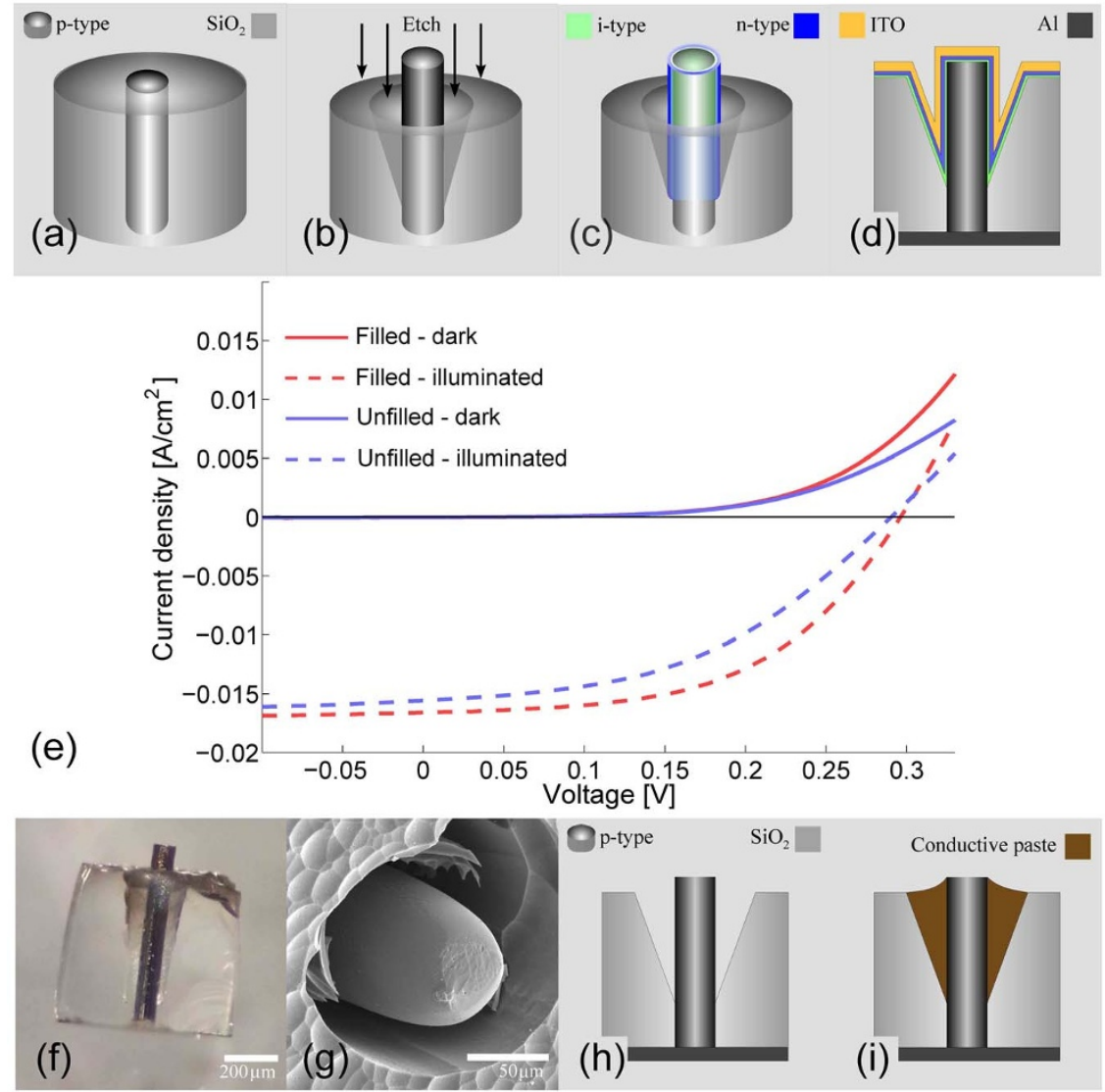

Figure 2 Stepwise solar cell fabrication procedure and solar cell performance. Processing of a single fibre segment into a solar device: $450 \mu \mathrm{m}$ segments (a) were selectively etched with HF from one side, resulting in the fibre core being mostly exposed (b) and (f) with a cavity around the fibre core due to the higher etch rate for $\mathrm{CaO}$ compared to $\mathrm{SiO}_{2}$. Deposition of a-i-Si and a-n-Si layers on top of the exposed wire using PECVD formed the p-i-n junction (c). A schematic representation (d) and an SEM image (g) of a processed solar cell and the dark and illuminated I-V curves (e) for a silicon wire with a diameter of $113 \mu \mathrm{m}$ are shown for both an as-made cell (h) and with the cavity filled with opaque conductive paste (i).

contact with the plasma likely resulted in thinner layers on the portions of the microwire within the etch cone around the fibre.

Light trapping simulations. The efficiency values measured above were based on an individual silicon fibre, using the cross-sectional area of the core in the efficiency calculation. However, in future devices, the silica surrounding the silicon core could contribute to absorption if the etch cone was coated with a reflective material and used as a light collector as shown in Fig. 4 a. In order to investigate the light trapping potential, ray-tracing simulations were performed, using unpolarised light, the optical properties of pure silicon for the core, and assuming an opaque silver coating on the cone walls for enhanced reflectivity. The structure was investigated for dependences on incident wavelengths, angle of incidence, the silicon core diameter and the silicon fill fraction. The silicon fill fraction, $\mathrm{F}$ is equal to $\left(\mathrm{D} / \mathrm{D}_{\mathrm{tot}}\right)^{2}$, where $\mathrm{D}$ is the diameter of the core, and $\mathrm{D}_{\text {tot }}$ is the outer diameter of the silica cone. Although the simulations were performed for individual fibres with their cladding, hexagonal close packing of fibres would allow realization of similar absorption values for large arrays.

The spectral absorption characteristics for wavelengths between $400 \mathrm{~nm}$ and $1100 \mathrm{~nm}$ are shown in Fig. 4b for several sets of cell parameters, with the calculated absorption of planar silicon shown for comparison. The geometry for the largest structure is similar to the devices we fabricated and measured. All designs perform quite well across the solar spectrum. Due to the high absorption at short wavelengths, only the spectral region above $800 \mathrm{~nm}$ had a significant dependence on the design parameters. The angular dependence of the $900 \mathrm{~nm}$ absorption for the same structures are shown in Fig. 4c along with spatial maps of the absorption for two different incidence angles $(\varphi)$ for the largest structure. Despite the simulation being performed for a poorly absorbed wavelength, it can be seen that the light trapping structure, with low silicon content, would have absorption superior to that of planar silicon over a wide range of angles. The smallest cores perform less well at large $\varphi$ because of the shallower cone angle, $\theta_{\mathrm{SiO}_{2}}$ and less absorption during each transmission pass through the silicon. The combination allows more light to escape the etch cone.

Fig. $4 \mathrm{~d}$ shows the results as the diameter of the core was varied between 10 and $100 \mu \mathrm{m}$ for several silicon fill fractions and fibre height equal $125 \mu \mathrm{m}$. A wavelength of $900 \mathrm{~nm}$ was used, as this is where the absorption length in silicon equals the height of the pillar, and conventional cells lose efficiency. For the lowest fill fractions, there is a rolloff in absorption for large diameters because the assumption of fixed height leads to a very shallow cone angle (large $\left.\theta_{\mathrm{SiO}_{2}}\right)$. Taller structures permit better light gathering for large cores at low fill fractions, but increase the total volume of silicon required. For a fill fraction greater than $15 \%$, there was little change in the calculated absorption over the core diameter range of 30-120 $\mu \mathrm{m}$.

\section{Discussion}

The dark and light current-voltage characteristics of the sample single-wire cell with the etch cone filled with opaque conductive paste, shown in Fig. 2, yield an overall conversion efficiency of $3.62 \%$. Measurement of the same cell without the paste, (contacting the ITO on the glass), gave similar open-circuit voltage, $\mathrm{V}_{\mathrm{oc}}$ and shortcircuit current density $\mathrm{J}_{\mathrm{sc}}$ values, but with a fill factor of 0.43 due to 


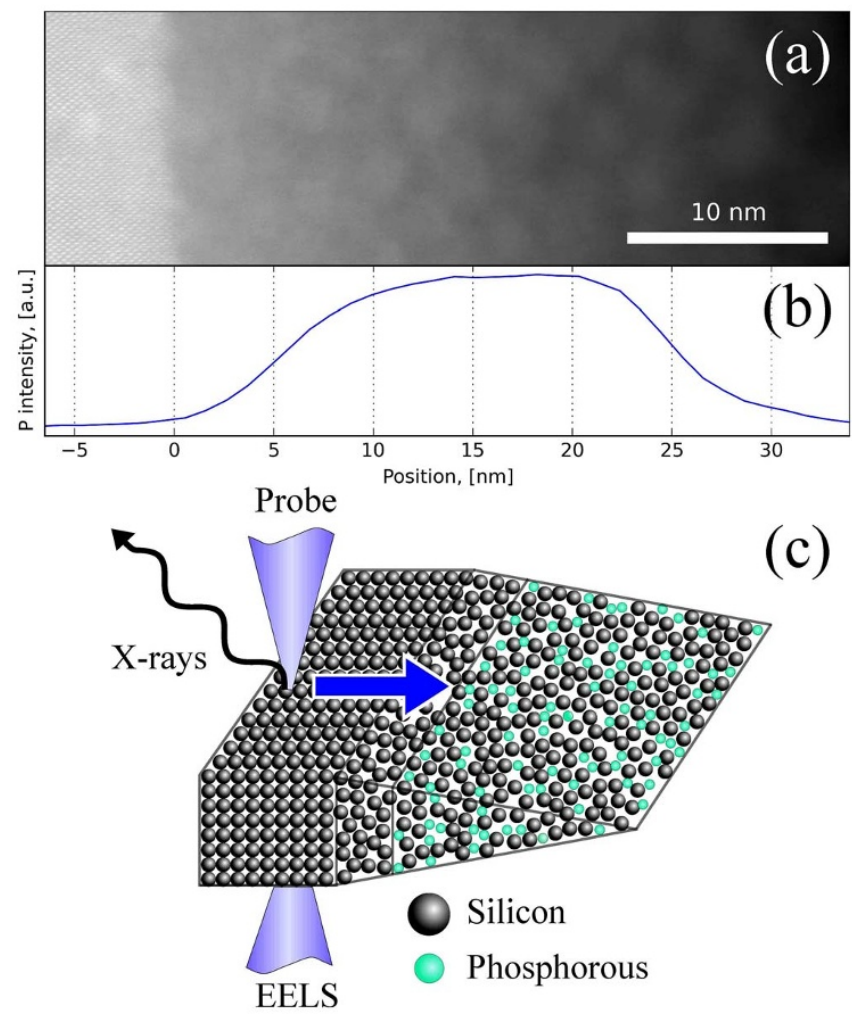

Figure 3 TEM analysis of the amorphous thin film. A scanning transmission electron micrograph of the amorphous silicon film on a crystalline $<100>$ silicon wafer with the viewing direction along the [011]axis of the wafer. The film thickness can be observed to be $\sim 32 \mathrm{~nm}$ with slight variations along the interface. The phosphorous signal (b) from EDS is offset from the crystalline interface, indicating the approximate thickness of the intrinsic and doped layers. Relative phosphorous content was found using EDS, normalized with respect to thickness using low loss electron energy loss spectroscopy. The loss of contrast and phosphorous signal towards the outer edge of the film is due to a thickness gradient from the specimen preparation, as shown schematically in (c).

reduced carrier extraction, because the indium tin oxide coating is thinner within the cavity. No reflective coating was applied inside the etch cone for these measurements, and it can be seen from comparing the short circuit current densities that the etch cone does not contribute to any increased light trapping for this cell. Optimization of the etched structure together with application of a reflective coating on the inside of the cone would be necessary to fully exploit the structure.

Fitting the dark characteristics of a single fibre cell (filled case) to the non- ideal diode equation

$$
I=I_{L}-\left[\exp \left(\frac{q V}{n k T}\right)-1\right],
$$

(where $I_{L}$ is the light-generated current, and $n$ is the ideality factor) between 0 and $0.35 \mathrm{~V}$ yields $n=1.77 \pm 0.11$, indicating preferred recombination at the surface and at the junction rather than in the crystalline quasi-neutral region. This supports the suggestion that the purity of the crystalline silicon fibres is higher than that of the upgraded metallurgical grade (UMG) quality of the starting material, likely due to a segregation based purification during melting and drawing of silicon into the fibre ${ }^{24}$. Similar ideality factor values have been reported for other radial junction solar devices ${ }^{9,14,15,17,25}$, typically attributed to their high surface to volume ratio.

The saturation current density was found to be $1200 \mathrm{nA} \mathrm{cm}{ }^{-2}$, which is high compared to reported values for similar designs ${ }^{15,17}$.
This high surface recombination is likely to be the main reason for the low observed open circuit voltage and thus improvements in junction- and surface quality are expected to improve the overall performance of the cell. The high net carrier recombination rate of the device presented here may be due to surface damage near the top of the fibre from the slicing process (Fig. 2f). An improved cutting technique and surface etch together with an optimization of the device layer thicknesses would likely increase the performance of the cell. The fibre radius could also be tuned to better match the minority carrier diffusion length ${ }^{21}$.

Simulations on the silver-coated reflective etch cone in the $\mathrm{SiO}_{2}$ (Fig. 5a) indicate that light gathering by these structures would be highly effective, with $>90 \%$ absorption for most geometries and wavelengths. This implies that device design could be optimized within wide limits based on the carrier diffusion length and surface recombination properties of the silicon cores. The spectrally averaged absorption of the cone plus fibre structure can be expected to exceed $90 \%$ at normal incidence, with little angular dependence up to $\sim 20$ degrees, for fill fractions of silicon $\leq 10 \%$. This is close to the absorption coefficient achieved for antireflection-coated texturized planar solar cells (95-97\%) ${ }^{38}$ which incorporate 10 times as much silicon, and require purification, bulk recrystallization and wafering. The simulations above do not assume any treatment to reduce reflection from the top of the silicon core, but for $10 \%$ fill fraction of silicon, this is responsible for $\sim 4 \%$ loss, or nearly half of the total reflection losses. Texturizing or antireflection coating of the top surface of the microwires would further increase the absorption.

A potential route for making practical large-scale devices is through assembly and slicing of bundles of fibres with subsequent processing of the entire slices into solar cells. This would result in the structure shown in Fig. 5b where the fibres are packed together hexagonally with a 0.91 packing factor. The imperfect packing would result in an additional loss over the simulated values, for which integration was carried out only to the edge of the cylindrical silica cladding. However, fusing the glass fibres, or fabricating multicore fibres in the drawing process would allow the etching process to be carried out until the etch cones merged, as shown in Fig. $5 c$, and absorption values would increase towards those for the simulated individual cladding-fibre structures. Future improvements in the junctions and single-wire conversion efficiency should carry over to array performance.

Potential fibre draw speeds of tens of meters per second are possible. With a 50-core parallel structure a $10 \%$ area fill fraction of silicon, and $125 \mu \mathrm{m}$ thickness cells (allowing a $50 \mu \mathrm{m}$ kerf loss during slicing), one tower could produce adequate material for $\sim 60$ square meters of solar cells per hour. This process would eliminate the time and energy consuming production of ingots, as the silicon is refined in situ as part of the drawing process, and would require only a tenth of the starting silicon material needed for a conventional cell, per unit area. It should be noted that, while an optical fibre tower was employed for these prototype fibres, there is no need for the high precision afforded by this equipment.

In conclusion we have demonstrated the feasibility of producing solar cells from low-grade silicon incorporated into glass-clad fibres made using the molten-core fibre drawing technique. The first single-fibre cells have efficiencies up to $3.62 \%$, with junction quality rather than silicon quality being the limiting factor, leaving room for improvements over the presented prototype. Future directions may include improvement of the processing of the silicon cores, utilization of the etch cone for light gathering and fabrication of multicore fibres ${ }^{28}$ for parallel production of cell arrays.

\section{Methods}

Fibre production. Glass preforms for the fibre-drawing process were prepared by coating the inside of a thick-walled silica tube with calcium hydroxide, drying the coating, then loading a solid silicon rod into the closed tube. The $\mathrm{CaO}$ coating was produced following the method of Nordstrand, et al. ${ }^{29}$ For this work, a $2 \mathrm{~mm}$ 
(a)
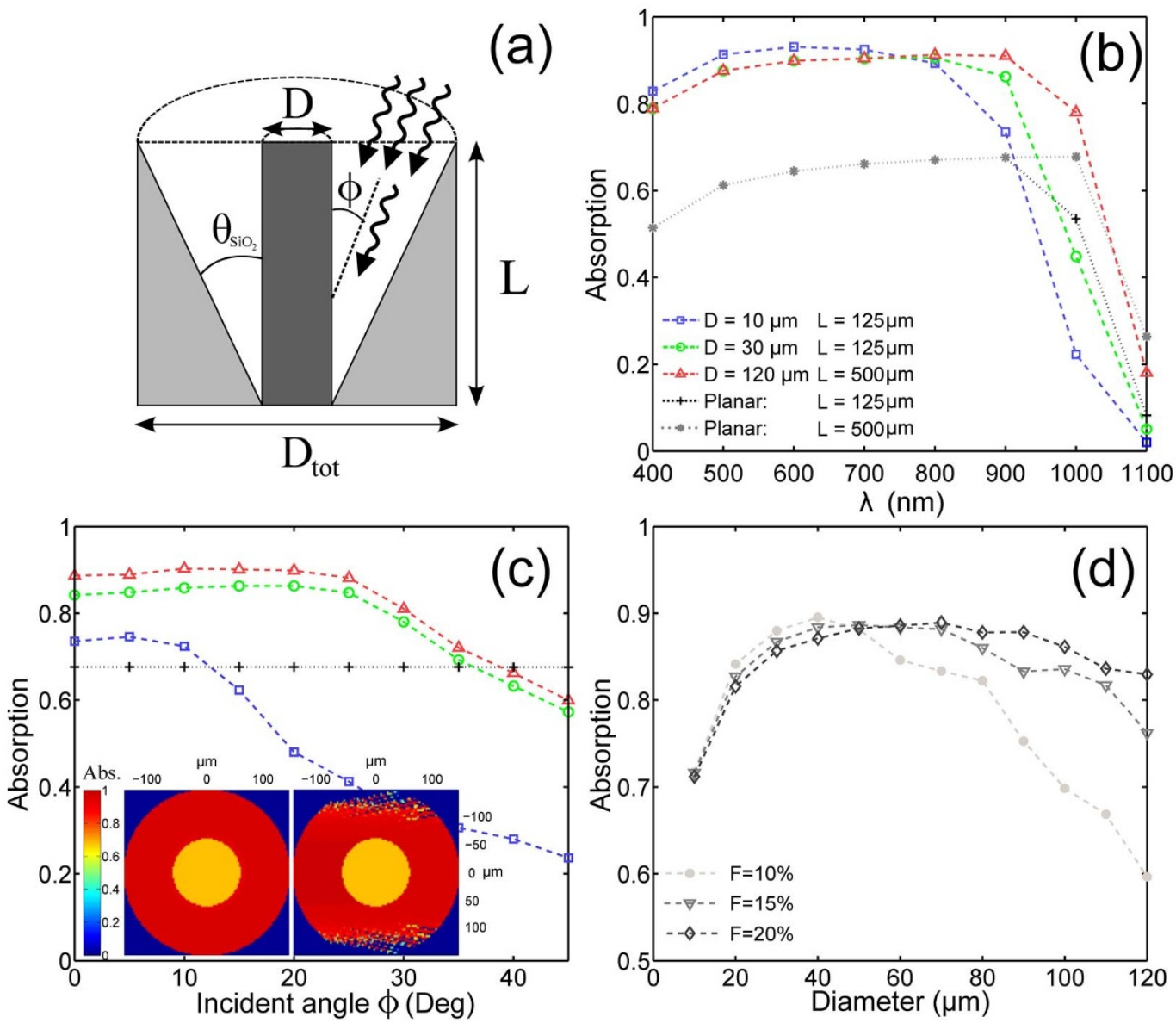

Figure $4 \mid$ Simulations of light gathering. (a) schematic of the light trapping structure, (b) absorption as a function of wavelength for three diameter and height combinations keeping $\theta_{\mathrm{SiO}_{2}}=10^{\circ}$. (c) absorption as a function of angle for $\lambda=900 \mathrm{~nm}$ and the same geometries used in (b) [the legend in (b) applies to (b) and (c)], and (d) absorption for three fill fractions as a function of diameter for $\lambda=900 \mathrm{~nm}$ and $\mathrm{L}=125 \mu \mathrm{m}$. Inset in (c) shows a spatial map of the absorption at normal incidence $(\varphi=0)$ and $\varphi=30$ degrees.

diameter p-type silicon rod of upgraded metallurgical grade (UMG 99.98\%) quality was inserted into a $\mathrm{CaO}(99.9 \%)$ coated $3 \times 30 \mathrm{~mm}$ silica glass tube, which then was drawn in a Heathway draw tower at a feed rate of $3 \mathrm{~mm} / \mathrm{min}$ and a draw rate of $2.7 \mathrm{~m} /$ min at $1950^{\circ} \mathrm{C}$ to form bulk lengths of fibre. Silicon core diameters between $50-$ $200 \mu \mathrm{m}$ were realized with the above parameters yielding an approximate $100: 1$ ratio of silica to silicon by volume.

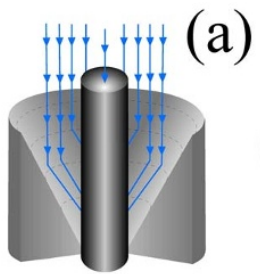

$\mathbf{S i}$

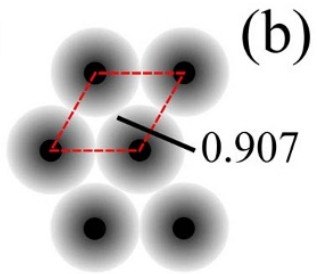

(c) $\mathrm{SiO}_{2}$

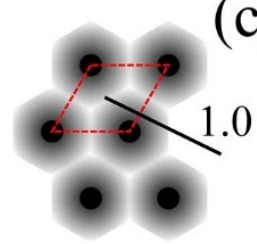

Figure 5 | Schematics illustrating utilization of the etch cone for scale-up purposes. (a) schematic of light gathering in a single etched silica-clad fibre and (b) top view of a bundle of fibres etched to gather light incident on the coated silica. There is a $9.3 \%$ loss due to air gaps for bundled cylinders, but fusing of the silica (c) would reduce or eliminate this loss. Packing factors are shown for cylinders and hexagonal columns.
Fibre characterization. For electrical characterization purposes, the silica cladding was etched away from the as-made fibres using an aqueous solution of $48 \% \mathrm{HF}$ $(27.6 \mathrm{M})$. The cores remained intact during this etch. The fibres were subsequently contacted with aluminium using a sputter coater and a shadow mask, and the resistivity was measured using the four-point probe technique. Crystallinity was confirmed by electron backscattered diffraction (EBSD) in a Hitachi SU-6600 field emission gun (FEG)-SEM operating at $20 \mathrm{keV}$. Grain lengths on the order of several $\mathrm{mm}$ were routinely observed. In order to investigate in-diffusion of oxygen during the fibre drawing process, the oxygen level of the source silicon was compared to that of an as-drawn fibre using secondary ion mass spectroscopy (SIMS). The investigation was conducted using a Cameca IMS 7 SIMS operating at $10 \mathrm{keV}$ with a primary beam of $\mathrm{Cs}^{+}$-ions, and the measured signals were averaged over a depth of $500 \mathrm{~nm}$ after sputtering through the surface contamination on the samples.

Device fabrication. The fabrication of the HIT structure and hence a functional solar cell was carried out by casting a bundle of the as-made fibres in epoxy for easier handling, and cutting this structure into $450 \mu \mathrm{m}$ slices, resulting in slices containing fibre segments like the one schematically illustrated in Fig. 2a. All processing was performed on the multifibre slices, but these were disassembled at the end of the fabrication cycle and single fibres were measured as solar cells. The segments were, subsequent to cutting, selectively etched from one side in a $48 \% \mathrm{HF}$ aqueous solution $(27.6 \mathrm{M})$ for $20 \mathrm{~min}$, revealing the front part of the fibre core and leaving the back untouched (Fig. 2b, f). This etch produces a conical cavity around the fibre due to the greater reactivity of the calcium- rich interface layer compared to that of $\mathrm{SiO}_{2}$, as seen in Fig. 2b,f. The exposed silicon cores were then etched for up to one minute in a modified CP4 solution consisting of a $7: 2: 1$ mixture of $65 \% \mathrm{HNO}_{3}$ in water (14.5 M), $48 \% \mathrm{HF}$ in water $(27.6 \mathrm{M})$ and $100 \% \mathrm{CH} 3 \mathrm{COOH}(17.4 \mathrm{M})$ in order to reduce surface irregularities as well as the saw-damaged areas of the fibre tips. The latter etch also resulted in the fibre core being shortened and rounded, as can be seen in Fig. 2g. The deposition of the amorphous silicon layers and hence the formation of the $\mathrm{p}-\mathrm{i}-\mathrm{n}$ junction was performed using an Oxford Instruments plasma enhanced chemical vapour deposition (PECVD) unit, operating at $150^{\circ} \mathrm{C}$. The temperature was chosen in accordance with Wang, et al. ${ }^{39}$, in order to minimize the formation of microcrystallites that are reported to cause disruptions in the localized band structure 
of the amorphous silicon film and an associated reduction of the passivation effectiveness. Prior to deposition, the fibre slices were given a 2 minute etch in a $5 \%$ HF aqueous solution $(2.9 \mathrm{M})$ to remove any native oxide that had formed after the $\mathrm{CP} 4$ etch, and to promote hydrogen termination of the fibres, enhancing surface passivation. The amorphous silicon was deposited at a rate of $0.36 \mathrm{~nm} / \mathrm{s}$ with a gas pressure of $1000 \mathrm{mTorr}$, a flow rate of $25 \mathrm{sccm} \mathrm{SiH}_{4}$ in $475 \mathrm{sccm} \mathrm{Ar}$ for the a-i-Si, and

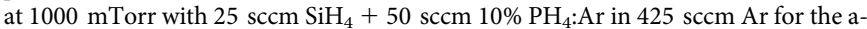
$\mathrm{n}$-Si. Finally, the contacts were sputter-deposited, applying $200 \mathrm{~nm}$ indium tin oxide (ITO, $\rho \sim 1.7 \times 10^{-3} \Omega-\mathrm{cm}, 1.2 \mathrm{~nm} / \mathrm{s}$ ) as the front contact and $150 \mathrm{~nm}$ aluminium $(1.3 \mathrm{~nm} / \mathrm{s})$ overcoated with $150 \mathrm{~nm}$ platinum $(1.66 \mathrm{~nm} / \mathrm{s})$ as the back contact.

Transmission electron microscopy. The analysis of the amorphous silicon film cross section was performed with high angle annular dark field scanning transmission electron microscopy (HAADF-STEM) using a JEOL ARM 200F TEM, and the thickness of the combined amorphous layers was measured to be $\sim 32 \mathrm{~nm}$ (Fig. 3a). The witness wafer was used due the challenge associated with TEM specimen preparation of fibres, but the film thicknesses on the wafer should be similar to that on the top surface of the fibre. Comparing the measured thickness with the deposition times used in the PECVD and assuming linear relationships between deposition time and thickness results in an a-i-Si layer of $\approx 7 \mathrm{~nm}$ and an a-n-Si layer of $\approx 25 \mathrm{~nm}$. The latter was confirmed by conducting an EDS-scan for phosphorous across the film cross-section, with thickness normalization performed using the simultaneously acquired low loss electron energy loss spectroscopy signal (Fig. 3b). Comparing the EDS scan with the corresponding STEM image reveals the predicted a-i-Si layer between the crystalline phase and the a-n-Si layer. A drop in the phosphorous signal close to the edge of the amorphous film is believed to be caused by a very poor signal to noise ratio caused by the reduced thickness of the sample when approaching the sample edge, as illustrated schematically in Fig. 3c.

Device testing. After disassembly of the slices used in the fabrication process, the IVcharacteristics for single fibre cells were measured under air mass 1.5 global (AM $1.5 \mathrm{G}$ ) illumination at normal incidence, and the cross-sectional area of the fibre core was used as the reference illuminated area. In addition to measuring the as-made cell, a measurement was performed where external influences from the glass surrounding the fibre core were eliminated by filling the groove surrounding the fibre core, visible in Fig. 2, with opaque conductive paste.

Optical modelling. A ray-tracing model was used to calculate the absorption of the idealized structure visible in Fig. 4a, for a variety of parameters. Using unpolarised light, the optical constants of silicon for the core and of silver for the cone surface, incoming rays were modelled through multiple reflections and transmission passes through the silicon until they were either absorbed or escaped the cone. Because it is metallized in the solar cell structure, the bottom surface of the silicon core was assumed, for simplicity, to have $\mathrm{R}=1$; this represents less than a $1 \%$ error at a $900 \mathrm{~nm}$ wavelength. For non-normal incidence, the problem was solved three-dimensionally by approximating the cone with 64 trapezoids, calculating the incidence angle and the reflection and transmission coefficients at each interface. The absorption coefficient of silicon was used with Beer's Law to calculate the absorption within the silicon core.

1. Holm, J. V. et al. Surface-passivated GaAsP single-nanowire solar cells exceeding 10\% efficiency grown on silicon. Nat. Commun. 4, 1498 (2013).

2. Law, M., Greene, L. E., Johnson, J. C., Saykally, R. \& Yang, P. Nanowire dyesensitized solar cells. Nat. Mater. 4, 455-459 (2005).

3. Wallentin, J. et al. InP nanowire array solar cells achieving $13.8 \%$ efficiency by exceeding the ray optics Limit. Science 339, 1057-1060 (2013).

4. Mohseni, P. K. et al. Monolithic III-V nanowire solar cells on graphene via direct van der Waals epitaxy. Adv. Mater. 26, 3755-3760 (2014).

5. Lee, M. R. et al. Solar power wires based on organic photovoltaic materials. Science 324, 232-235 (2009).

6. Fan, X., Huang, L., Du, J., Liu, Z. \& Tao, C. Role of interfacial strain in fiber-shaped solar cell based on TiO2 nanotube arrays. J. Nanosci. Nanotechnol. 14, 7111-7116 (2014).

7. Orf, N. D. et al. Fiber draw synthesis. Proc. Natl. Acad. Sci. U. S. A. 108, 4743-4747 (2011).

8. Toivola, M., Ferenets, M., Lund, P. \& Harlin, A. Photovoltaic fiber. Thin Solid Films 517, 2799-2802 (2009).

9. Kelzenberg, M. D. et al. Enhanced absorption and carrier collection in Si wire arrays for photovoltaic applications. Nat. Mater. 9, 239-244 (2010).

10. Tian, B. et al. Coaxial silicon nanowires as solar cells and nanoelectronic power sources. Nature 449, 885-889 (2007).

11. Seo, K. et al. Si microwire solar cells: improved efficiency with a conformal $\mathrm{SiO} 2$ layer. ACS Nano 7, 5539-5545 (2013).

12. Kendrick, C. E. et al. Radial junction silicon wire array solar cells fabricated by gold-catalyzed vapor-liquid-solid growth. Appl. Phys. Lett. 97, 143108 (2010).

13. Jia, G., Steglich, M., Sill, I. \& Falk, F. Core-shell heterojunction solar cells on silicon nanowire arrays. Sol. Energy Mater. Sol. Cells 96, 226-230 (2012).

14. Kelzenberg, M. D. et al. High-performance Si microwire photovoltaics. Energy Environ. Sci. 4, 866-871 (2011)
15. Kim, D. R., Lee, C. H., Rao, P. M., Cho, I. S. \& Zheng, X. Hybrid Si microwire and planar solar cells: Passivation and characterization. Nano Lett. 11, 2704-2708 (2011).

16. Gharghi, M., Fathi, E., Kante, B., Sivoththaman, S. \& Zhang, X. Heterojunction silicon microwire solar cells. Nano Lett. 12, 6278-6282 (2012).

17. Yoon, H. P. et al. Enhanced conversion efficiencies for pillar array solar cells fabricated from crystalline silicon with short minority carrier diffusion lengths. Appl. Phys. Lett. 96, 213503 (2010).

18. Guo, N. et al. Fabrication of silicon microwire arrays for photovoltaic applications. Appl. Phys. A 102, 109-114 (2011).

19. Garnett, E. \& Yang, P. Light Trapping in Silicon Nanowire Solar Cells. Nano Lett. 10, 1082-1087 (2010).

20. Dan, Y. et al. Dramatic reduction of surface recombination by in situ surface passivation of silicon nanowires. Nano Lett. 11, 2527-2532 (2011).

21. Kayes, B. M., Atwater, H. A. \& Lewis, N. S. Comparison of the device physics principles of planar and radial p-n junction nanorod solar cells. J. Appl. Phys. 97, 114302 (2005).

22. Gharghi, M. On the design and applicability of nanowire solar cells using lowgrade semiconductors. J. Appl. Phys. 111, 034501 (2012).

23. Levine, J. D. \& Jensen, M. J. Texas Instruments Inc., Production of semiconductor grade silicon spheres from metallurgical grade silicon particles. United States patent US 5,069,740 A. 1991 Des 3.

24. Martinsen, F. A., Nordstrand, E. F. \& Gibson, U. J. Purification of melt-spun metallurgical grade silicon micro-flakes through a multi-step segregation procedure. J. Cryst. Growth 363, 33-39 (2013).

25. He, R. et al. Silicon p-i-n junction fibers. Adv. Mater. 25, 1461-1467 (2013).

26. Ballato, J. et al. Silicon optical fiber. Opt. Express 16, 18675-18683 (2008).

27. Scott, B. L., Wang, K. \& Pickrell, G. Fabrication of n-type silicon optical fibers. IEEE Photonics Technol. Lett. 21, 1798-1800 (2009).

28. Gumennik, A. et al. Silicon-in-silica spheres via axial thermal gradient in-fibre capillary instabilities. Nat. Commun. 4 (2013)

29. Nordstrand, E. F., Dibbs, A. N., Eraker, A. J. \& Gibson, U. J. Alkaline oxide interface modifiers for silicon fiber production. Opt. Mater. Express 3, 651-657 (2013).

30. Gibson, U. J., Dibbs, A., Eraker, A., Hawkins, T. \& Ballato, J. Alkaline oxide modifiers for the production of semiconductor fibers. Workshop Spec. Opt. Fibers Their Appl. W1.3; DOI:10.1364/wsof.2013.w1.3 (2013)

31. Mishima, T., Taguchi, M., Sakata, H. \& Maruyama, E. Development status of high efficiency HIT solar cells. Sol. Energy Mater. Sol. Cells 95, 18-21 (2011).

32. Taguchi, M., Terakawa, A., Maruyama, E. \& Tanaka, M. Obtaining a higher V-oc in HIT cells. Prog. Photovolt. 13, 481-488 (2005).

33. Wang, Q. et al. Efficient heterojunction solar cells on p-type crystal silicon wafers. Appl. Phys. Lett. 96, 013507 (2010).

34. Dao, V. A. et al. Simulation and study of the influence of the buffer intrinsic layer, back-surface field, densities of interface defects, resistivity of p-type silicon substrate and transparent conductive oxide on heterojunction with intrinsic thinlayer (HIT) solar cell. Sol. Energy 84, 777-783 (2010).

35. Putnam, M. C. et al. Si microwire-array solar cells. Energy Environ. Sci. 3, 1037-1041 (2010).

36. Kanevce, A. \& Metzger, W. K. The role of amorphous silicon and tunneling in heterojunction with intrinsic thin layer (HIT) solar cells. J. Appl. Phys. 105, 094507 (2009).

37. Zhao, L., Zhou, C. L., Li, H. L., Diao, H. W. \& Wang, W. J. Design optimization of bifacial HIT solar cells on p-type silicon substrates by simulation. Sol. Energy Mater. Sol. Cells 92, 673-681 (2008).

38. Parretta, A. et al. Angle-dependent reflectance measurements on photovoltaic materials and solar cells. Opt. Commun. 172, 139-151 (1999).

39. Wang, T. H. et al. Effect of emitter deposition temperature on surface passivation in hot-wire chemical vapor deposited silicon heterojunction solar cells. Thin Solid Films 501, 284-287 (2006).

\section{Acknowledgments}

This work was financially supported by the Norwegian Research Council, the Norwegian Micro- and Nano-Fabrication Facility, NorFab (197411/V30), the infrastructure Grant 197405 (NORTEM) program of the Research Council of Norway, and the NTNU Discovery Program.

\section{Author contributions}

F.A.M. did all the experimental work with exception of the TEM-analysis conducted by M.N. and the fibre production conducted by T.H. and J.B.; B.K.S. performed the light trapping simulations. U.J.G. conceived and supervised the project. F.A.M. and U.J.G. organized and wrote the paper with all authors contributing to the analysis and discussion of the results.

\section{Additional information}

Competing financial interests: The authors declare no competing financial interests.

How to cite this article: Martinsen, F.A. et al. Silicon-core glass fibres as microwire radial-junction solar cells. Sci. Rep. 4, 6283; DOI:10.1038/srep06283 (2014). 

NoDerivs 4.0 International License. The images or other third party material in this article are included in the article's Creative Commons license, unless indicated otherwise in the credit line; if the material is not included under the Creative
Commons license, users will need to obtain permission from the license holder in order to reproduce the material. To view a copy of this license, visit http:// creativecommons.org/licenses/by-nc-nd/4.0/ 